\title{
Random Resistor Network Model of Minimal Conductivity in Graphene
}

\author{
Vadim V. Cheianov, ${ }^{1}$ Vladimir I. Fal'ko, ${ }^{1}$ Boris L. Altshuler, ${ }^{1,2,3}$ and Igor L. Aleiner ${ }^{2}$ \\ ${ }^{1}$ Physics Department, Lancaster University, Lancaster, LAI 4YB, United Kingdom \\ ${ }^{2}$ Physics Department, Columbia University, 538 West 120th Street, New York, New York 10027, USA \\ ${ }^{3}$ NEC-Laboratories America, Inc., 4 Independence Way, Princeton, New Jersey 085540, USA
}

(Received 20 June 2007; published 22 October 2007)

\begin{abstract}
Transport in undoped graphene is related to percolating current patterns in the networks of $n$ - and $p$-type regions reflecting the strong bipolar charge density fluctuations. Finite transparency of the $p-n$ junctions is vital in establishing the macroscopic conductivity. We propose a random resistor network model to analyze scaling dependencies of the conductance on the doping and disorder, the quantum magnetoresistance and the corresponding dephasing rate.
\end{abstract}

Graphene - an atomic monolayer of graphite [1] — is a gapless semiconductor with linear [2] electron spectrum. The carrier density in a graphene-based field effect transistor (GraFET) can be varied continuously, from $p$ type to $n$ type. Experimental results [1] are surprising: the resistance per square never exceeds several $\mathrm{k} \Omega$ 's in contrast to the pinch-off in conventional (gapfull) semiconductors. Such a behavior of GraFET persists over a broad temperature range $10^{-2} \mathrm{~K} \lesssim T \lesssim 10^{2} \mathrm{~K}$. The conductivity reaches its minimum when the gate-controlled carrier density, $n_{g}=0$,- "neutrality point." Similar observations were reported for the bilayer graphene [3].

Attempts to understand the finite value of the minimal conductivity in graphene have so far addressed the role of "chirality" of the Dirac-type quasiparticles and influence of various short-range defects on the quantum transport [4] in a homogeneous graphene sheet. However, there is an emerging evidence [5-7] that charge density in GraFETs is "mesoscopically" inhomogeneous-probably, reflecting the fluctuations of the charge trapped in the underlying substrate, or on its surface. An inhomogeneity of charge density in a "charge-neutral" graphene sheet, $n_{g}=0 \mathrm{im}$ plies that it can be viewed as a checkerboard of $n$ - and $p$-type doped regions separated by weakly conducting [8] $p$ - $n$ junctions. In this Letter we propose a random resistor network model for such a system and use it to describe classical and quantum transport.

The model of the random resistor network (RRN) is formulated on the square lattice with the lattice constant $a$ and sites labeled by integers $(i, j)$. Sites $(i, j),\left(i^{\prime}, j^{\prime}\right)$ with $\left|i^{\prime}-i\right|>1,\left|j^{\prime}-j\right|>1$ are not connected directly. Each pair of sites with $\left|i^{\prime}-i\right| \leq 1$, and $\left|j^{\prime}-j\right| \leq 1$, is connected by a link with the conductance $\mathbb{G}_{(i, j)}^{\left(i^{\prime}, j^{\prime}\right)}=\mathbb{G}_{\left(i^{\prime}, j^{\prime}\right)}^{(i, j)}$

$$
\begin{aligned}
\mathbb{G}_{(i, j)}^{(i+1, j+1)} & =g\left[1+(-1)^{i+j} \eta_{i, j}\right] / 2 ; \\
\mathbb{G}_{(i, j+1)}^{(i+1, j)} & =g\left[1-(-1)^{i+j} \eta_{i, j}\right] / 2 ; \\
\mathbb{G}_{(i, j)}^{(i+1, j)} & =\mathbb{G}_{(i, j)}^{(i, j+1)}=\gamma g, \quad \gamma \ll 1 .
\end{aligned}
$$

Here $\eta_{i, j}$ is a random variable,

$$
\eta_{i, j}= \pm 1, \quad\left\langle\eta_{i, j}\right\rangle=p,\left\langle\eta_{i, j} \eta_{k, l}\right\rangle=\delta_{i k} \delta_{j l},
$$

where $\langle\ldots\rangle$ means the ensemble averaging.

The relation of model (1) to graphene with inhomogeneous charge density, $n(r)=n_{g}+\delta n(\mathbf{r})$ is illustrated on Fig. 1. Assume that the density fluctuations $\delta n(\mathbf{r})$ are characterized by the length scale $a$ so that $\langle\delta n(\mathbf{r}) \delta n(\mathbf{r}+$ $\mathbf{x})\rangle=\delta n^{2} f(x / a) ; a$ may be determined by, e.g., the thickness of the insulating substrate. As long as $a^{2} \delta n \gg 1$, one can view the system as a combination of electron $(n)$ and hole $(p)$ puddles of the size of the order of $a$. Each puddle contains a large number of carriers, which are characterized by the Fermi wave vector $k_{F} \sim \sqrt{\pi \delta n} \gg a^{-1}$. If $i+j$ is even (odd), the site of the RRN corresponds to the $n(p)$ puddle and is marked by red (blue) color on Fig. 1. The correspondence between the puddles and the lattice sites encodes the fact that the observable conductivity is determined by the random links between the puddles rather than the local conductivity of a puddle.

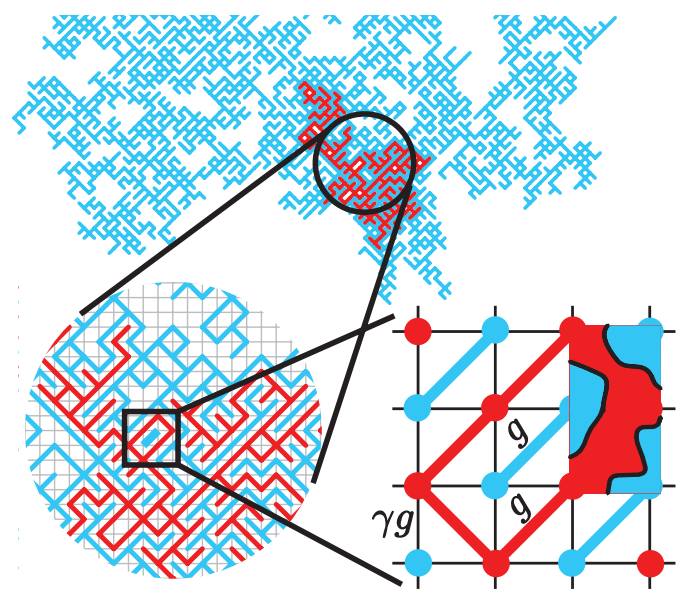

FIG. 1 (color). RRN representation of a graphene sheet with nominally zero doping, $n_{g}=0$. 
Each plaquette of the RRN, see the inset for Fig. 1, has one and only one diagonal connection-either $p-p$ or $n-n$ link. This is described by Eqs. (1a) and (1b). If parameter $p=0$, then $p-p$ and $n-n$ connections appear with equal probability, and $p>0(p<0)$ describe the electron (hole) doping. The boundary between the puddles has a finite, though small, transparency [8]. This transparency is characterized by $\gamma \ll 1$ in Eq. (1c) and depicted by the squarelattice grid on Fig. 1.

Scaling analysis. - First, let us consider a RRN with $p=0, \gamma=0$ in Eq. (1). Then, two-color random networks on a bipartite lattice have peculiar geometrical features illustrated in Fig. 1 by a computer-generated sample. Emerging patterns are typical for the percolation theory [9]. The RRN is critical; i.e., the geometry of the RRN is self-similar on all length scales: the $L \times L$ network contains, typically, a larger cluster of one polarity (such as the red cluster in the middle part of Fig. 1) which separates a pair of smaller blue clusters. Each of those clusters is in turn a shell for several smaller red clusters, etc. Such alternating cluster embedding represents a scale-invariant property of an infinite network. As a result, larger and larger parts of the network become excluded from the monocolor percolation upon the increase of the size $L$. Therefore, the observable conductance $G(L)$ decreases with the increase of $L$. The corresponding critical behavior of its mean value is $[9,10]$

$$
\langle G(L)\rangle \sim(a / L)^{x} g ; \quad x \approx 0.97 .
$$

This means that the conductivity is not defined.

Finite $p$ and $\gamma$ are relevant perturbations for the percolation leading to a finite correlation length $\xi(p, \gamma)$. At $L \gg$ $\xi$, the RRN is not critical and consists of independent patches of size $\xi$. Thus, the conductivity is finite

$$
\sigma(\gamma, p) \equiv\langle G(L \rightarrow \infty)\rangle \sim[a / \xi(p, \gamma)]^{x} g .
$$

Because of the scale invariance, $\xi$ depends on $p, \gamma$ as

$$
\xi(p, \gamma \rightarrow 0) \sim a|p|^{-\nu} ; \quad \xi(p \rightarrow 0, \gamma) \sim a \gamma^{-\mu} .
$$

Its $p$ dependence is the dependence of the correlation length on deviations from the percolation threshold [9]:

$$
\nu=4 / 3 .
$$

To the best of our knowledge, the scaling of $\xi$ with $\gamma$ was not considered in literature, yet. We found

$$
\mu=1 /(h+x) \approx 0.37,
$$

where $x$ is the conductance exponent, Eq. (2), and $h=7 / 4$ (see Ref. [9]) is the exponent of the cluster outer perimeter $\mathcal{P}(L) \simeq a(L / a)^{h}$ for the cluster of the size $L$.

To derive Eq. (4c), consider the red cluster of the size $L$ embedded into the conducting blue cluster. Small but finite $\gamma$ does not affect the criticality if the leak through the perimeter of this cluster $\mathcal{P}$ is much smaller than the conductance of the blue cluster, the latter is also of the order of $G(L)$. One can use Eqs. (2) and (1c) to write this condition as $(a / L)^{x} \gtrsim(L / a)^{h} \gamma$. This yields $L \lesssim \xi=a / \gamma^{\mu}$, with $\mu$ given by Eq. (4c). For $L>\xi$, the leakage through the cluster boundary is efficient enough for RRN to become uniform.

The dependence of the conductivity on $p, \gamma \neq 0$, is

$$
\xi(p, \gamma) \sim a \gamma^{-\mu} / \mathcal{F}\left(p / p^{*}\right), \quad p^{*}=\gamma^{\mu / \nu},
$$

where $\mathcal{F}$ is a universal scaling function. The form of Eq. (5) is protected by the scale invariance. The numerics described below are well fit by the interpolation formula

$$
\mathcal{F}(z)=\left(1+z^{2}\right)^{\nu / 2},
$$

reproducing both $z \ll 1$ and $z \gg 1$ asymptotic behavior. Substituting Eqs. (5) and (6) into Eq. (3) we find

$$
\sigma(\gamma, p)=u g \gamma^{\alpha} \mathcal{F}^{x}\left(p / p_{*}\right), \quad \alpha=x \mu \approx 0.36,
$$

where $u \approx 1.3$ is a coefficient found from the fit of the numerical data. Equation (7) completely describes nonanalytic dependence of the conductivity on both the leakage parameter $\gamma \ll 1$ [11] and adjustable doping $p$. Recently, an attempt was made in Ref. [12] to explain the graphene minimal conductivity using a mean field theory. We believe that Ref. [12] correctly describes the limit of high carrier density, where the fluctuations of local conductivity are small, but fails near the neutrality point where the percolation physics start to dominate.

Numerical simulations of model (1) were performed on square lattices with $L \leq 500 a$. Numerical evaluation of the network conductance has been done using the bus-bar boundary conditions at the two opposite edges along the direction of the current flow and the periodic boundary conditions in the orthogonal direction. Realizations of the RRN were generated in families spanning the interval $p \in$ $[-1 ; 1]$ of the network parameter, in steps of $\Delta p=$ 0.0125 . Each family was obtained from the regular network at $p=1$ (containing only blue links) by sequentially replacing $L^{2} / \Delta p$ blue links by red ones in randomly chosen plaquettes. Averaging of the data has been performed over 300 families. To minimize the finite-size effects we (i) identified $1 / L$ corrections by finite-size scaling from $L=50 a$ to $L=500 a$; and (ii) subtracted those corrections by means of numerical extrapolation. Numerical results summarized on Fig. 2 are in excellent agreement with the scaling formulas (6) and (7).

Sample-to-sample fluctuations. - Geometry of the percolation cluster is nontrivial. In particular, its linear size depends on the particular realization and reconnecting only few bottleneck links may change it substantially. Therefore, the conductance $G(L)$ is a random quantity, which is characterized by its distribution function $P(G)$ rather than only by its average. Scaling invariance of the critical cluster at $L \ll \xi(p, \gamma)$ constrains the functional form of this distribution to $P(G)=\langle G\rangle^{-1} \mathbb{P}(G /\langle G\rangle)$, $\int \mathbb{P}(x) d x=1$, where $\mathbb{P}(x)$ is a universal function. It implies the variance 

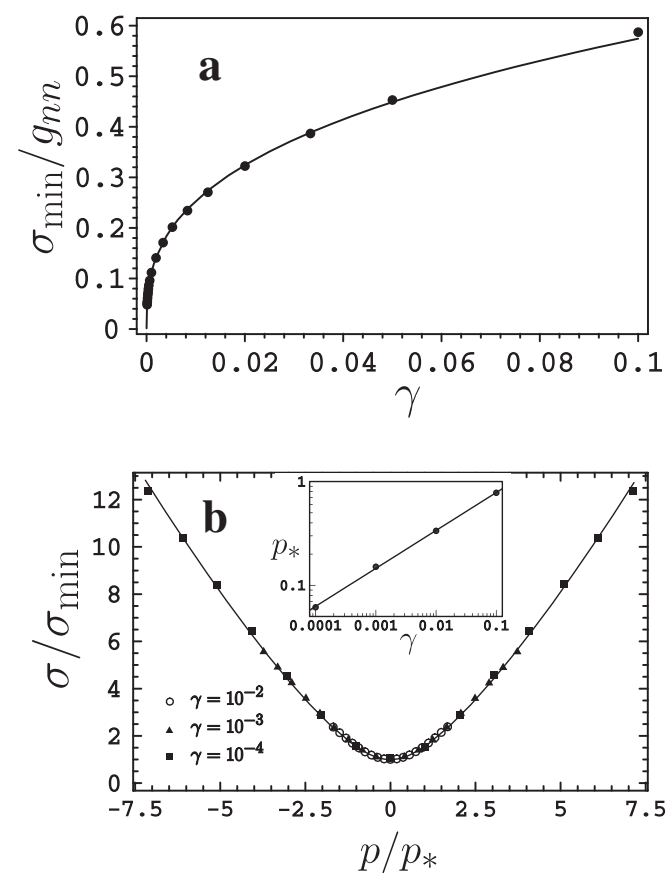

FIG. 2. (a) Conductivity $\sigma_{\min }(\gamma) \equiv \sigma(\gamma, p=0)$. Numerical data are represented by dots. The solid line is the best fit to the scaling law in Eq. (7). (b) Collapse of the conductivity data obtained for RNNs with various $-1 / 2<p<1 / 2$ and values of the parameter $\gamma$ onto a single curve, Eq. (6), represented by solid line. For each value of $\gamma$ the corresponding values of the parameter $p_{*}$ were found using by fitting $\sigma(p)$ to Eqs. (6) and (7); see inset.

$$
\left\langle\delta G^{2}(L \ll \xi)\right\rangle=u_{2}\langle G(L \ll \xi)\rangle^{2},
$$

where $u_{2} \simeq 1$ is a universal numerical coefficient. For $L \gg \xi$, the central limit theorem is restored, and we find

$$
\left\langle\delta G^{2}(L \gg \xi)\right\rangle=\left\langle\delta G^{2}(\xi)\right\rangle(\xi / L)^{2}=u_{2} \sigma^{2} *(\xi / L)^{2} .
$$

Figure 3(a) illustrates the fluctuations of RRN conductance. One can see that not only value of the minimal conductance but also its position fluctuates from sample to sample. Figure 3(b) shows an excellent agreement with the scaling form (9) for $p=0$.

The fluctuations (9) are self-averaging. Notice, however, that because $\xi$ is large, those fluctuations can exceed the universal conductance fluctuations $(\mathrm{UCF}) \simeq e^{2} / \hbar[13]$ which are not self-averaging as long as the phase coherence is preserved. The scale for the energy dependence of fluctuations (9) is determined by the properties of the individual bottleneck links in the cluster and significantly exceeds the Thouless energy for the UCF. This observation is consistent with numerical findings of Ref. [14].

Quantum magnetoresistance. - Until now we ignored the quantum interference and interaction effects. Some, and most importantly those that give rise to the quantum magnetoresistance [15], can be analyzed within the percolation cluster framework [16]. The role of the weak localization (WL) is to renormalize the conductance of each
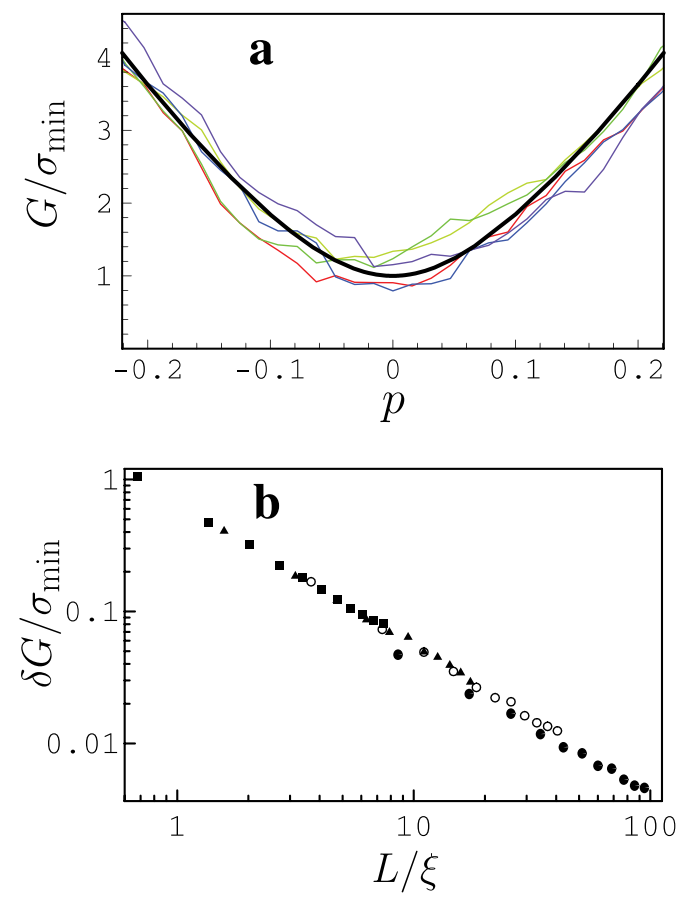

FIG. 3 (color online). (a) Conductance of several $100 \times 100$ samples with $\gamma=10^{-4}$ as a function of $p$ : The parameter $p$ is gradually changed from -0.2 to 0.2 by flipping a small number of randomly chosen links from $p$ - $p$ type to the $n-n$ type. The fluctuations of the conductance are the strongest at $p=0$. (b) rms of sample-to-sample fluctuations of RRN conductance for $p=0$ and various values of $\gamma$ [legend is the same as in Fig. 2(b)] as a function of system size, $L$.

link in Eq. (1) due to the interference of the random walk paths that go through the same link twice. For WL magnetoresistance only long paths are important. Thus, one can assume that the return probability is independent of each particular links, so that it can be included into the renormalization of the conductance $g$.

As usual [15], the evaluation of the WL correction consists in finding the classical return probability for the random walk, whereas the quantum mechanics determines the relevant spatial scales and the prefactors in this problem, which are the same as in the case of homogeneous graphene [17].

To find the probability for the returning path, one poses the problem of the diffusion on the lattice, Eq. (1), where the link conductances $\mathbb{G}$ are replaced by the diffusances. Then, comparison with Eqs. (2) and (3) yields the scaledependent diffusion coefficient $D(L)$ in terms of the observable diffusion constant at large distances, $D_{\xi}$ :

$$
D(L) \sim[\xi / \min (L, \xi)]^{x} D_{\xi}
$$

Our treatment of the WL differs from that of Ref. [17] only by the scale dependence of the diffusion constant. The magnetoconductance $\delta \sigma(B)=\sigma(B)-\sigma(0)$ in not-so-low magnetic field $B$ can be estimated as 


$$
\begin{gathered}
\delta \sigma \approx \frac{e^{2}}{\pi^{2} \hbar} \sum_{J=0}^{1}(-1)^{J} \sum_{M=-J}^{J} \int_{0}^{\sqrt{e B / \hbar c}} \frac{q d q D_{\xi}}{D(1 / q) q^{2}+\Gamma_{J}^{M}}, \\
\Gamma_{0}^{0}=\frac{1}{\tau_{\phi}} ; \quad \Gamma_{1}^{M}=\frac{1}{\tau_{\phi}}+\frac{1}{1+|M|}\left(\frac{1}{\tau_{\perp}}+\frac{|M|}{\tau_{\|}}\right) .
\end{gathered}
$$

Here $\tau_{\perp}$ is the intervalley scattering time and $1 / \tau_{\|}$is the rate for intravalley scatterings breaking certain symmetries of the system, see Ref. [17] for more details. The phase relaxation time $\tau_{\phi}$ will be estimated below.

If $\tau_{\phi} \gg \xi^{2} / D_{\xi}$, Eq. (11) reduces to the results of Ref. [17]. The specifics of the scale-dependent diffusion (10) are revealed in the opposite limit $\tau_{\phi} \ll \xi^{2} / D_{\xi}$ :

$$
\begin{aligned}
\delta \sigma & \approx \frac{e^{2}}{\pi^{2} \hbar} \sum_{J=0}^{1}(-1)^{J} \sum_{M=-J}^{J}\left(\frac{\mathcal{L}_{J, M}}{\xi}\right)^{x} \tilde{Y}\left(\frac{e B \mathcal{L}_{J, M}^{2}}{c \hbar}\right) ; \\
\mathcal{L}_{J, M} & =\xi\left[D_{\xi} /\left(\xi^{2} \Gamma_{J}^{M}\right)\right]^{1 /(2+x)},
\end{aligned}
$$

where $\tilde{Y}(z)$ is a universal function [18] with the asymptotic behavior $\tilde{Y}(z \ll 1) \simeq z^{2}$, and $\tilde{Y}(z \gg 1) \approx 1-z^{-x / 2}$. The overall magnetoresistance (12) is strongly suppressed in comparison with that for the homogeneous graphene, $|\delta \sigma| \ll e^{2} /\left(\pi^{2} \hbar\right)$.

The phase relaxation in ordinary disordered conductors is dominated by the electron-electron interaction [19]. The time defining the magnetoresistance curvature $\partial^{2} \sigma /\left.\partial B^{2}\right|_{B \rightarrow 0}$ is controlled by the dimensionless conductance $G(L)=2 \pi \hbar G(L) / e^{2}$ :

$$
\hbar / \tau_{\phi} \simeq T / G\left(L_{\phi}\right) ; \quad L_{\phi}=\left(D \tau_{\phi}\right)^{1 / 2},
$$

where $L_{\phi} \equiv \mathcal{L}_{0,0}$ is the dephasing length. As all the geometric properties of the system in Eq. (13) are encoded into the scale dependences of $G(L)$ and $D(L)$, Eq. (13) is valid even for diffusion along the critical cluster. We obtain from Eqs. (2), (3), (10), and (13)

$$
L_{\phi}=\xi \Phi\left(T_{\xi} / T\right) ; \quad T_{\xi}=\hbar G(\xi) D_{\xi} / \xi^{2} \propto \xi^{-2(1+x)},
$$

where $\Phi(z)$ is a scaling function with the asymptotic behavior $\Phi(z \gg 1) \approx \sqrt{z}, \Phi(z \ll 1) \approx z^{1 /(2+2 x)}$. Condition $T \sim T_{\xi}$ determines the crossover from the critical to the normal diffusion: $L_{\phi}\left(T>T_{\xi}\right)>\xi, L_{\phi}\left(T<T_{\xi}\right)<\xi$.

Equations (12) and (14) predict interesting doping behavior of the magnetoresistance at fixed temperature. At $p \lesssim 1, \xi(p)$ is small, $T_{\xi}>T$ and the usual logarithmic magnetoresistance occurs. For $p \rightarrow 0, T_{\xi}<T$, and the WL becomes suppressed as $1 / \xi^{x}$. Such prediction seems to be consistent with the experiment of Ref. [20].

Relation to the experimental parameters. - To relate Eq. (7) to the properties of graphene, we have to connect the parameters $\gamma$ and $g$ with the physical parameters of the sample. If the charge inhomogeneity is the dominant disorder in the graphene monolayer, we can estimate the conductance of $n-n(p-p)$ connections between puddles as $g \sim \frac{e^{2}}{\hbar} a k_{F}$ and conductance of the $p$ - $n$ junction separat- ing puddles of the opposite polarity as $\gamma g \sim \frac{e^{2}}{\hbar}\left(a k_{F}\right)^{1 / 2}$; see Eq. (2) of Ref. [8], $\gamma \sim\left(a k_{F}\right)^{-1 / 2} \sim\left(a^{2} \delta n\right)^{-1 / 4}$. According to Eq. (7) we then estimate $\sigma_{\min }=\sigma(\gamma, p=$ $0) \sim \frac{e^{2}}{\hbar}\left(a^{2} \delta n\right)^{(1 / 2)-(\alpha / 4)} \sim \frac{e^{2}}{\hbar}\left(a^{2} \delta n\right)^{0.41}$.

In conclusion, we constructed a random resistor network model, which adequately takes into account strong fluctuations of the local charge density and, thus, of the local conductivity of the mono- and bilayer graphene near neutrality point. This model describes inhomogeneous current percolating through the system, giving rise to the scaling dependencies of the observable conductivity on the doping and disorder. Quantum magnetoresistance and the sampleto-sample fluctuations are analyzed.

We acknowledge discussions with A.K. Geim and P. Kim and support from EPSRC and ESF-FoNE SpiCo.

[1] K.S. Novoselov et al., Science 306, 666 (2004); A. K. Geim and K. S. Novoselov, Nat. Mater. 6, 183 (2007).

[2] P. R. Wallace, Phys. Rev. 71, 622 (1947).

[3] K. S. Novoselov et al., Nature Phys. 2, 177 (2006).

[4] J.H. Bardarson et al., arXiv:0705.0886, and references therein.

[5] J. Martin et al., arXiv:0705.2180.

[6] S. Cho and M.S. Fuhrer, arXiv:0705.3239.

[7] To the best of our knowledge, the importance of "mesoscopic" inhomogeneities for the transport in graphene was first highlighted in M. I. Katsnelson, K. S. Novoselov, and A. K. Geim, Nature Phys. 2, 620 (2006).

[8] V. Cheianov and V. Fal'ko, Phys. Rev. B 74, 041403 (2006).

[9] M. B. Isichenko, Rev. Mod. Phys. 64, 961 (1992) and references therein.

[10] Index $x$ has been determined using the ratio of two wellestablished percolation theory indices listed on pages $972-$ 974 of Ref. [9]: those describing scaling of the conductivity and correlation length as functions of density, $p$ (note, that in the proposed RRN of minimal conductivity in graphene, $p_{c}=0$ ).

[11] For $\gamma \gtrsim 1$ the correlation length $\xi \simeq a$, and one obtains a trivial result $\sigma=\gamma g$.

[12] S. Adam et al., arXiv:0705.1540.

[13] Mesoscopic Effects in Solids, edited by B.L. Altshuler, P. A. Lee, and R. Webb (North-Holland, Amsterdam, 1992).

[14] A. Rycerz, J. Tworzydlo, and C.W. J. Beenakker, arXiv:cond-mat/0612446.

[15] B. L. Altshuler et al., Phys. Rev. B 22, 5142 (1980).

[16] D. E. Khmel'nitskii, JETP Lett. 32, 229 (1980).

[17] E. McCann et al., Phys. Rev. Lett. 97, 146805 (2006); I. L. Aleiner and K. B. Efetov, ibid. 97, 236801 (2006).

[18] Estimate (11) reproduces $\tilde{Y}(z \gg 1)$. At small magnetic field, $z \ll 1$, Eq. (11) cannot be used.

[19] I. L. Aleiner, B.L. Altshuler, and M.E. Gershenson, Waves Random Media 9, 201 (1999), and references therein.

[20] S. V. Morozov et al., Phys. Rev. Lett. 97, 016801 (2006). 